\title{
Degrees as nominalized properties: Evidence from differential verbal comparatives in Mandarin Chinese ${ }^{1}$
}

Qiongpeng Luo - Nanjing University

Zhiguo Xie- The Ohio State University

\begin{abstract}
Whether degrees should be modeled as simple semantic primitives or ontologically complex entities has been an issue in recent formal semantic research. This article aims to make a contribution to this scholarly enterprise by investigating the Differential Verbal Comparative (DVC) construction in Chinese. DVCs exhibit peculiar properties : (i) obligatory differentials, and (ii) DPs as differentials(e.g., liang ben xiaoshuo 'two CL novel'). We propose that a degree is the entity correlate of a property that is formed on the basis of a measure, akin to Chierchia-style kind. This new kind of degree, coupled with a difference function-based semantics for comparatives, correctly predicts the behaviors of DVCs which would otherwise remain formally inscrutable. This article's contributions are twofold: (i) it provides direct support for the degree-as-kind analysis by extending its empirical scope; and (ii) by combining degrees as kinds with a difference function-based semantics, it represents an improvement over the previous degree-as-kind analysis based on linear ordering.
\end{abstract}

Keywords: comparatives, degrees, kinds, Mandarin Chinese, differential verbal comparatives.

\section{Introduction}

Over the past several decades, there has been a significant amount of discussion on what exactly degrees are. Approaches to this question roughly fall within two schools, which bear distinct (though not completely incompatible) consequences for the semantics of comparatives.:

(1) Two approaches to degrees: primitive vs. complex

(i) The standard approach: Degrees are semantic primitives formalized as points or intervals on an abstract scale, akin to real numbers (cf., Seuren, 1973; von Stechow, 1984; Heim, 1985; Kennedy, 1999; Schwarzschild and Wilkinson, 2002; Kennedy and McNally, 2005; Kennedy, 2007; Beck, 2012);

(ii) The not-so-standard approach: Degrees are not semantic primitives, but rather ontologically complex entities. Research within this approach treats degrees as equivalence classes (Cresswell, 1976), as tropes (Moltmann, 2009), oras kinds (Anderson and Morzycki, 2015; Scontras, 2017). The interested reader can also refer to Grosu and Landman (1998) and Castroviejo and Schwager (2008) for relevant discussion.

There are many unresolved issues on this topic. For example, do all comparatives make use of the same kind of degree?If not, is it possible for some comparative constructions to make

\footnotetext{
${ }^{1}$ We would like to thank Curt Anderson, Thomas Grano, Miao-Ling Hsieh, Chris Kennedy, Xiao Li, , and Yafei Li for constructive comments on an earlier draft of the paper. We are also indebted to Manfred Krifka, Louse McNally, Stephanie Solt, and other scholars in the audience of Sinn und Bedeutung 22 for inspiring suggestions and comments. This work is financially supported by National Social Science Foundation of China (NSSFC) under grant \# 16BYY006 to Qiongpeng Luo.
} 
use of degrees as points, while other comparative constructions make use of degrees as kinds? Is there any empirical evidence for the degree-as-kind vs. degree-as-point dichotomy? If so, what regulates between them?

These issues get more complicated in the face of data from Mandarin Chinese. Recently, one case of within-language variation among comparative constructions in Mandarin Chinese has been identified and intensively studied: comparative constructions making use of degree ordering along some scale vs. comparative constructions making use of direct comparison of two sets of individuals with no reference to, or mediation by, degrees. While comparative constructions such as the $b i$ adjectival comparative (AC) and the transitive comparative have been argued to represent the first comparison strategy (Xiang, 2005; Lin, 2009; Grano and Kennedy, 2012), Li (2009, 2015a) takes the differential verbal comparatives (DVCs) in Mandarin Chinese to exemplify what she calls "degreeless comparison", which in her analysisinvolves one-to-one mapping between two sets of individuals. The degree-based comparison vs. degreeless comparison is exemplified by the examples in (1)-(2), respectively:

(2) Degree-based comparatives: $b i$ adjectival comparatives (ACs)

Zhangsan bi Lisi gao (san gongfen).

Zhangsan BI Lisi tall (three centimeters) ${ }^{2}$

'Zhangsan is (three centimeters) taller than Lisi.' Lin (2009); Grano and Kennedy (2012)

(3) Degreeless comparatives: differential verbal comparatives (DVCs)

Zhangsan bi Lisi duo du-le *(liang ben xiaoshuo).

Zhangsan BI Lisi DUO read-ASP two CL novels

'Zhangsan's reading exceeded Li's reading by two novels.'

DVCs () differ from ACs in two respects: (i) differentials in DVCs are obligatory, while differentials in ACs are optional, and (ii) differentials in DVCs can take the form of DP, e.g., liang ben xiashuo 'two CL novels', while differentials in ACs can only be measure phrases (MPs), e.g., san gongfen 'three centimeters'. These two peculiarities of DVCs stand out and challenge the standard semantics of degrees and DPs.

This article aims to offer a motivated explanation for the seemingly inscrutable properties of DVCs: Why do DVCs allow DP-like differentials? Why are differentials obligatory in DVCs? Setting in a broader cross-linguistic context, we note that DPs -- and their close cousins, relatives clauses (RCs) -- denoting degrees are widely attested across languages. In light of recent studies on gradability and comparison (especially Anderson and Morzycki, 2015; Scontras, 2017), we motivate an analysis that treats degrees as equivalence classes (Cresswell, 1976), or Chierchia-style quantity- and quality-uniform properties (Chierchia, 1998; McNally, 2001; Scontras, 2017). However, adopting a degree-as-kind analysis does not tackle all the problems raised by DVCs. One standing issue has to do with the semantic composition: since kinds, unlike points, are not linearly ordered, the compositionality becomes a non-trivial issue in this degree-as-kind analysis. To fix this problem, we take a revisionist strategy. On the one

\footnotetext{
${ }^{2}$ Abbreviations are as follows: ASP: aspectual markers; BI: $b i$ (a marker to introduce the standard of comparison); CL: classifiers; DE: modification marker de; DEM: demonstratives; DUO: duo. To eliminate controversy, in this article, we gloss duo simply as DUO, although it has been glossed either as 'more' or 'many/much' in the literature.
} 
hand,e follow the most recent works such as Anderson and Morzycki (2015) to treat degrees as kinds. On the other hand, we discard their semantics for comparatives based on linear ordering. Instead, we adopt a difference function-based semantics for comparatives. We demonstrate that this new semantics that combines degree-as-kind with difference functions not only correctly predicts the behaviors of DVC sentences, but desirably circumvents the problems faced by Anderson and Morzycki (2015).

\section{Differential Verbal Comparatives in Mandarin Chinese}

A typical DVC sentence comprises four components: (i) a target of comparison ( $\mathrm{DP}_{1}$ ), (ii) an optional standard-of-comparison phrase introduced by the morpheme bi ([bi $\left.\mathrm{DP}_{2}\right]$ ), (iii) a verb introduced by duo or shao, and (iv) an obligatory differential phrase, as exemplified by liang-ben xiaoshuo 'two CL novels' in (4b).

(4) a. $\mathrm{DP}_{1}\left(\right.$ bi $\left.\mathrm{DP}_{2}\right) d u o /$ shao $\mathrm{V} *($ differential phrase)

b. Zhangsan bi Lisi duo du-le liang ben xiaoshuo

Zhangsan BI Lisi DUO read-ASP two CL novel

'Zhangsan read two more novels than Lisi did.'

At least three features merit further discussion. First, although as shown in (4b), some DVC sentences can be translated into amount comparatives in English, ${ }^{3}$ DVCs and amount comparatives are by no means alike. Suppose, for instance, both Zhangsan and Lisi went for shopping, Zhangsan bought one cellphone and one Surface Pro, Lisi only bought one Surface Pro. The following sentence in (5), taking the form of DVC, can be felicitously used to describe this situation, while the same situation cannot be felicitously expressed by amount comparative in English::

(5) a. Situation: Zhangsan bought a cellphone and a Surface Pro, Lisi only bought a Surface Pro:

b. Zhangsan bi Lisi duo mai-le shouji.

Zhangsan BI Lisi DUO buy-ASP cellphone

$\checkmark$ 'Zhangsan bought one more thing than Lisi, which is cellphone.'

x 'Zhangsan bought more cellphone than Lisi.'

Second, besides regularMPs such as san mi 'three meters', san gongjin 'three kilos', differential phrases in DVCs can take almost all forms of DPs: an indefinite DP, a kinddenoting term (realized as bare nouns in Chinese), and even a proper name, as illustrated by (6a-c), respectively:

(6) a. Differential phrase $=$ indefinite DP:

Zhangsan bi Lisi duo du-le liang ben xiaoshuo

Zhangsan BI Lisi DUO read-ASP two CL novel

'Zhangsan read two more novels than Lisi did.'

b. Differential phrase = kind-denoting term:

Zhangsan bi Lisi duo mai-le shouji.

Zhangsan BI Lisi DUO buy-ASP cellphone

${ }^{3}$ For more about amount comparatives, see Morzycki 2016 (Ch. 6) and references therein. 
'Zhangsan bought one more thing than Lisi, which was a cellphone.'

c. Differential phrase = proper name:

Zhangsan bi Lisi duo qu-le New York.

Zhangsan BI Lisi DUO go-ASP New York

'Zhangsan went one more place than Lisi, which was New York.'

By contrast, for ACs, differential phrases can only take the form of MP:

(7) Zhangsan bi Lisi gao \{liang limi /*liang ben shu $\}$..

Zhangsan BI Lisi tall two centimeters/ two CL books

'Zhangsan is $\{$ two centimeters/*two books\} taller than Lisi.'

Third, as pointed out by $\mathrm{Li}(2015 \mathrm{a})$, unlike $b i \mathrm{ACs}$, differentials in DVCs are obligatory.

The differences between DVCs andACs can be summarized as follows:

Table 1: A variation between comparatives in Chinese: DVCs vs. ACs

\begin{tabular}{cccccc}
\hline & $\begin{array}{c}\text { Standard } \\
\text { marker }\end{array}$ & $\begin{array}{c}\text { Predicates of } \\
\text { comparison }\end{array}$ & $\begin{array}{c}\text { Obligatory } \\
\text { differentials }\end{array}$ & $\begin{array}{c}\text { MP } \\
\text { differential } \\
\text { s }\end{array}$ & $\begin{array}{c}\text { DP } \\
\text { differentials }\end{array}$ \\
\hline ACs & $b i$ & Gradable adjectives & - & + & - \\
DVCs & $b i$ & Duo/shao $+\mathrm{V}$ & + & + & + \\
\hline
\end{tabular}

Obviously, DVCs pose non-trivial challenges for both the standard semantics of degrees (as points on a scale) and DPs (presumably referring to mere individuals). Here is a Comparative Puzzle in Chinese:

(8) A Comparative Puzzle

(i) If both DVCs and ACs are comparisons of degrees, then we are forced to accept the conclusion that DPs have the same denotations as MPs, i.e., both refer to degrees, contracting the standard view that DPs refer to individuals.

(ii) If DVCs are fundamentally different from ACs, then we miss a unified account of comparatives, and we are unable to explain the commonalities between them, for example, why both take the comparative form and involve the same standard marker bi.

In the literature, $\mathrm{Li}(2009,2015 \mathrm{a})$ is the first serious attempt to provide a detailed empirical description and semantic analysis of DVCs. Before presenting our account, a critical reviewof her analysis is in order.

\section{The previous analysis}

\subsection{Li's (2015) mapping-based account of DVCs}

Li's analysis of DVCs is largely based on two assumptions: (i) when the verb following duo/shao is transitive or di-transitive, the differential DP in the DVC sentence is individualdenoting DP, which presumably does not denote a degree; and (ii) different from (direct or 
indirect) comparison of degrees, comparison of two sets of individuals calls for mapping between two sets, rather than degree ordering. ${ }^{4}$ According to this analysis, the predication of the verb on the subject $\left(\mathrm{DP}_{1}\right)$ yields a set $\mathrm{A}$, and the predication of the verb on the standardof-comparison phrase $\left(\mathrm{DP}_{2}\right)$ yields another set $\mathrm{B}$. Duo establishes a mapping relation whereby every element in set $\mathrm{B}$ is paired with a unique and different element in set $\mathrm{A}$. The differential DP denotes the subset of set A whose elements are left unpaired with those in set B. Formally, Li defines the semantics of $d u o$ as in (9a), where "O" is an overlap relation.

(9) a. $\llbracket d \mathrm{duo}_{\mathrm{f}} \rrbracket^{\mathrm{g}}=$

$$
\lambda \mathrm{P}_{<\mathrm{e},<\mathrm{e}>>} \lambda \mathrm{k}_{\mathrm{e}} \lambda \mathrm{y}_{\mathrm{e}} \lambda \mathrm{x}_{\mathrm{e}} \cdot \forall \mathrm{z}_{\mathrm{e}}\left[\mathrm{P}(\mathrm{z})(\mathrm{y}) \rightarrow \exists \mathrm{t}_{\mathrm{e}}[\mathrm{t}=\mathrm{g}(f)(\mathrm{z}) \wedge \mathrm{PROPER}(\mathrm{g}(\mathrm{f})) \wedge \mathrm{P}(\mathrm{t})(\mathrm{x}) \wedge \mathrm{P}(\mathrm{k})(\mathrm{x}) \wedge \neg \mathrm{t} \mathrm{ok}]\right]
$$

b. PROPER is a function (of type $<<\mathrm{e}, \mathrm{e}>, \mathrm{t}>$ ) which is true of $\mathrm{g}(f)$ iff $\mathrm{g}(f)$ preserves the taxonomic level introduced by the differential phrase.

The definition of duo in the DVC construction requires four semantic arguments: (i) a twoplace predicate $P$ corresponding to the verb $\mathrm{V}$ in (4a), (ii) an individual $k$ corresponding to the differential phrase, (iii) an individual $y$ corresponding to $\mathrm{DP}_{2}$, and (iv) an individual $x$ corresponding to $\mathrm{DP}_{1}$. The function $f$ in the definition is a mapping function, whose value is assigned by the assignment function $g$. This definition involves a mapping between two sets of individuals to which $\mathrm{DP}_{1}$ and $\mathrm{DP}_{2}$ relate by way of the predication as specified by $\mathrm{V}$. It identifies the difference between the two sets with the denotation of the differential phrase. In addition, Li's analysis requires, by way of a PROPER function as defined in (9b), that all the individuals under mapping be of the same taxonomic sort/level. The taxonomic information is specified by the differential phrase.

The LF structure that Li assumes for the above semantics of duo is given in (10) ( $\mathrm{Li}(2015 \mathrm{a})$ : Ex. (48)). On this account, the morpheme $b i$ projects a PP, and it is semantically vacuous. The standard-of-comparison phrase is a simple PP complement. It does not have any clausal syntactic structure, and does not denote a degree argument. The semantic interpretation of the sentence is spelled out in (11). It states that for each novel read by Lisi, Zhangsan read a matching copy, and that there are two novels that Zhangsan read but for which Lisi did not read matching copies.

(10) [s [liang ben xiaoshuo [s $\lambda \mathrm{i}$ [vp [DP Zhangsan] [vp [pp bi Lisi] [vp [v duo du-le] ti] $\ldots$

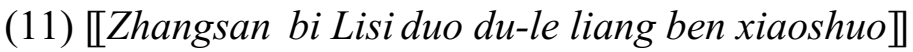

$=\exists \mathrm{x}_{\mathrm{e}}\left[\operatorname{novel}(\mathrm{x}) \wedge \# \mathrm{x} \geq 2 \wedge \forall \mathrm{z}_{\mathrm{e}}[\operatorname{read}(\mathrm{z})(\right.$ Lisi $)$

$\rightarrow \exists \mathrm{t}_{\mathrm{e}}[\mathrm{t}=\mathrm{g}(f)(\mathrm{z}) \wedge \operatorname{PROPER}(\mathrm{g}(f)) \wedge \operatorname{read}(\mathrm{t})($ Zhangsan $) \wedge \operatorname{read}(\mathrm{x})($ Zhangsan $\left.\left.) \wedge \neg \mathrm{tox}]\right]\right]$

Li (2009, 2015a) claims that compared to a degree semantic account, her degreeless, mapping-based approach to the DVC construction fares better in accommodating important differences observed between the bi ACs and the DVC. First, as shown above, duo and shao are the only two elements that can be used right before a verb to form a DVC sentence, and other gradable adjectives or adverbs cannot ((12), repeated from 63(a-b) in Li (2015a)). Li's

\footnotetext{
${ }^{4} \mathrm{Li}(2009,2015 \mathrm{a})$ takes $d u o$ to be ambiguous depending upon the argument structure of the verb and, relatedly, the lexical category of the differential phrase. For the sake of simplicity, our primary focus in this paper is on cases where the verb following duo/shao is a transitive verb and where the differential phrase is not a measure phrase or factor phrase.
} 
explanation is that gradable adjectives and adverbs like kuai 'fast' and renzhen 'attentively' are standardly analyzed to involve relations between individuals and degrees. She argues that the difference in semantic type disallowss gradable predicates other than duo and shao in the DVC construction.

$\begin{array}{rllllll}\text { (12) a. *Zhangsan } & \text { bi } & \text { Lisi } & \text { kuai } & \text { pao-le } & \text { liang } & \text { gongli. } \\ \text { Zhangsan } & \text { BI } & \text { Lisi } & \text { fast } & \text { run-ASP } & \text { two kilometer } \\ \text { b. *Zhangsan } & \text { bi } & \text { Lisi } & \text { renzhen } & \text { du-le } & \text { liang ben shu. } \\ \text { Zhanagsan } & \text { BI } & \text { Lisi } & \text { attentively } & \text { read-ASP } & \text { two CL books }\end{array}$

Second, recall that a differential phrase is required in a DVC sentence, whereas it is optional in a $b i \mathrm{AC}$ sentence. On Li's mapping-based account, in addition to denoting the relevant difference, the differential phrase is needed in the DVC so as to mark the taxonomic sort/level whereby mapping is done. According to Li's analysis, without a differential phrase, mapping would be random and baseless. For degree comparison, the dimension along which comparison is performed comes from the gradable predicate, and no separate taxonomic information is required for the comparison to be meaningful.

\subsection{Problems with Li's degreeless analysis}

Li's analysis is motivated by two assumptions: (i) differential DPs DVCs are semantically akin to genuine individual-denoting DPs occurring in non-comparative contexts; (ii) DPs do not denote degrees. We demonstrate that both of the assumptions are challenged by empirical data.

Differential DPs in DVCs are by no means like DPs in non-comparative contexts. Evidence comes from two observations: (a) pronominalization: a differential DP in a DVC sentence cannot be referred back to by individual-denoting pronouns or empty categories, but can be referred back to by a degree/kind anaphor; (b) topicalization: differential DPs in DVCs cannot be topicalized, unlike genuine individual-denoting DPs in non-comparative contexts. Given these two empirical observations, Li's evidence for treating differential DPs as individual-denoting does not warrant the conclusion that she intends for.

First, if differential DPs in DVC sentences have exactly the same semantics as genuine individual-denoting DPs occurring in non-comparative contexts, we should expect that they can be referred back to by pronouns or empty categories. This prediction is not borne out: (13) is at best marginally acceptable and stands in stark contrast with the perfectly acceptable sentence in (14).

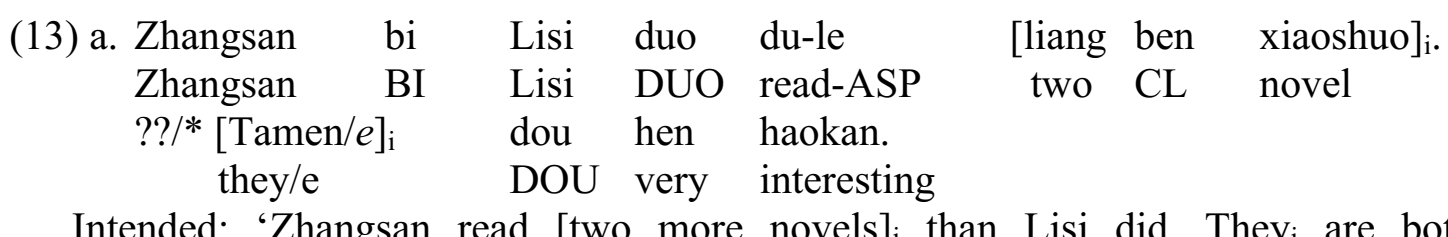

Intended: 'Zhangsan read [two more novels] $]_{i}$ than Lisi did. They $y_{i}$ are both very interesting.'
b. Zhangsan
bi
Lisi duo du-le
[liang ben xiaoshuo $]_{i}$. 


\section{Zhangsan BI Lisi DUO read-ASP two CL novel \\ ??/*Wangwu ye duo du-le [tamen] . \\ Wangwu also DUO read-ASP them} too.'

Intended: 'Zhangsan read two more novels than Lisi did. And Wangwu read them,

(14) Zhangsan jintian mai-le

Zhangsan today buy-ASP

[liang ben xiaoshuo $]_{\mathrm{i}}$.

$[\text { Tamen } / e]_{i}$

two CL novel

they/e

dou hen haokan

'Zhangsan bought [two novels] $]_{i}$ today. They $y_{i}$ are very interesting.'

By contrast, degree anaphors like zheme "such (this)" and na'me "such (that)" can be used to refer to differential DPs in DVC sentences.

(15)

$\begin{array}{lllllll}\text { Zhangsan } & \text { bi } & \text { Lisi duo du-le } & {[\text { liang ben }} & \text { xiaoshuo }]_{i} . \\ \text { Zhangsan } & \text { BI } & \text { Lisi DUO read-ASP } & \text { two } & \text { CL novel } \\ \text { Wangwu } & \text { ye duo du-le } & \text { [na'me } & \text { duo]. } & \\ \text { Wangwu } & \text { also DUO read-ASP that many } & \\ \text { 'Zhangsan read two more novels than Lisi did. Wangwu read these two novels } \\ \text { /that many more, too.' }\end{array}$

Second, topicalization provides another compelling piece of evidence that differential DPs in DVCs are not individual-denoting. It has been widely accepted that topics in Mandarin Chinese are subject to a definiteness constraint (Chao, 1968; Li and Thompson, 1981). When this constraint is met, a DP can be topicalized, as shown in (16) below:

(16) a. Zhangsan du-le Jane Eyre.

Zhangsan read-ASP Jane Eyre

b. [Topic Jane Eyre], Zhangsan du-le $e$.

If differential DPs in DVCs were indeed parallel to DPs in non-comparative contexts, then they should be able to be topicalized, provided that the definiteness constraint is satisfied. This prediction is not borne out, again. Even when differential DPs in DVC sentences take the form of proper names or demonstrative phrases, they normally cannot be topicalized:

(17) a. Zhangsan bi Lisi duo du-le zhe ben xiaoshuo.

Zhangsan BI Lisi DUO read-ASP Dem CL novel

b. */?? [Topic Zhe ben xiaoshuo], Zhangsan bi Lisi duo du-le $e$.

As a further note, genuine degree expressions cannot be topicalized in Mandarin Chinese:

(18) a. Zhangsan bi Lisi gao liang limi.

Zhangsan BI Lisi tall two centimeters

'Zhangsan is taller than Lisi by 2 centimeters.'

b. *Liang limi, Zhangsan bi Lisi gao. 
Based on the above evidence, we postitulate that differential DPs in DVC sentences still involve degrees, not individuals alone. (19) below is another natural example demonstrating that differential DPs in DVC sentences denote degrees. In the context of talking about paper product consumption, (19) can be understood to mean that paper consumption in America exceeds that in China by the amount of xylem fiber worth of the forest in question can produce, not the physical forest itself.

(19) Meiguo yi nian yao bi zhongguo duo xiaohao yan-qian zhe pian senlin. US one year will BI China DUO consume eye-before this CL forest 'The US will consume the-forest-before-us-worth more (paper) than China in one year.

Our observation that differential DPs in DVC sentences involve degree semantics actually reconfirmations the long-held view that DPs can have a degree component. Grosu and Landman (1998: 132) cites the English example in (20) (originally due to Heim (1987)) whose most natural reading is about drinking the same amount of champagne as was spilled, though one can imagine a stretched situation in which people, like curly dogs, are licking up the ground.

(20) It will take us the rest of our lives to drink [DP the champagne that they spilled that evening].

Furthermore, degree-denoting DPs are widely attested in many languages, most of them are typologically unrelated to Mandarin Chinese. Rett (2014) reports a number of cases in English in which DPs denote degrees, not individuals (21) (see also Cresswell, 1976). The Romanian example (22), repeated from Rett's (15) (due to Grosu (2009)), illustrates the same pattern. According to Grosu, the gap associated with the wh-phrase cat "is the internal argument of a predicate that selects degrees (on a scale that the predicate specifies)"'. Degreedenoting DPs are also found in Hindi-Urdu, which employs a correlative (which takes the form of a DP) to convey comparison between two degrees. (23) is from Bhatt and Takahashi (2011: 593). Degree-denoting DPs taking the form of relative clauses are also attested in Japanese, a language geographically close but genetically unrelated to Mandarin Chinese. According to Sudo (2015), the complement of yori in (24) should be analyzed as a relative clause headed by a covert element that denotes a degree.

(21) English ( Rett 2014)

a. Four pizzas is more than we need. [degree interpretation]

b. Many guests is several more than Bill anticipated. [degree interpretation]

(22) Romanian (Grosu 2009)

(Cele) nouă kilograme cât cântăreste bagajul tău de mână nu

DEM nine kilos how-much weighs luggage-the your of hand not te vor împiedica să te urci in avion you will-PL prevent SUBJ REFL climb-1SG in plane plane.'

'[DP (The) nine kilos that your handbag weighs] won't prevent you from boarding the

(23) Hindu-Urdu (Bhatt and Takahashi 2011) 
[Pim-ne kal jitnii kitaabe parh-i:] [Tina-ne aaj

Pim-Erg yesterday how.many.f books.f read-Pfv. FPl Tina-Erg today

us-se zyaadaa kitaabe parh-i:].

that-than more books read-Pfv.FPl

Lit.: 'How many books Pim read yesterday, Tina read more books than that today.'

(24) Japanese (Sudo 2015)

John-wa [[ Bill-ga katta ]-yori ] takusan hon-o katta

John-top Bill-nom bought-than many book-acc bought

'John bought more books than the amount of books that Bill bought.'

In this section, we have demonstrated that the assumptions motivating Li's degreeless analysis of DVCs are unwarranted. Nextwe will provide an account that takes degrees to be individual correlates of properties (i.e., nominalized properties) to accommodate the observed facts in relation to DVCs.

\section{Toward a new kind of degree}

In the standard degree-based framework, degrees are "abstract representation of measurement", modeled as points along an abstract scale, akin to real numbers (Seuren, 1973; von Stechow, 1984; Schwarzschild and Wilkinson, 2002; Kennedy and McNally, 2005; Beck, 2012; Morzycki, 2016). ${ }^{5}$ We agree with Li (2009, 2015a) that DVCs in Mandarin Chinese, which allow DPs to function as differential phrases, pose non-trivial challenges for this degree-as-point analysis. At the same time, as we have shown, Li's alternative degreeless analysis relying on the one-to-one mapping between two sets also runs into difficulty. An adequate account of the DVC facts calls for a reconsideration of the ontology of degrees.

An early alternative approach to the degree-as-point analysis can be traced back to Cresswell (1976), which places degrees in the model, but does not treat them as primitives. Cresswell analyzes the plural count noun men as at times denoting " $x$ is a set of men" and at other times denoting " $\mathrm{x}$ is a $y$-membered set of men", where $y$ is a variable over cardinalities (pp. 277278). He defines degrees as equivalence classes, viz., groups of individuals that are the same with respect to some measure ( a particular gradable property) such as weight and height. (p. 281). 180 centimeters, for instance, is the class of pairs of a world $w$ and an individual $x$ such that individual $x$ is 180 centimeters tall in world $w$ (Castroviejo and Schwager, 2008).

Cresswell's seminal idea ushered in an approach that adopts a richer ontology of degrees. For example, Grosu and Landman (1998) treat degrees as tuples of an individual, a property, and a measure, Moltmann (2009) takes degrees to be tropes, Anderson and Morzycki (2015) argue for a deep connection between degrees and kinds. Most recently, Scontras (2017) studies the degree noun amount in English and proposes that a degree is an individual correlate of a property that is formed on the basis of some measure. Consider:

(25) a. I ate that amount of apples every day for a year.

\footnotetext{
${ }^{5}$ An alternative view is to taking degrees as intervals (Wilkinson and Schwarzschild 2002). It should be noted that taking degrees as intervals does not circumvent the challenges posed by DVCs, since both degree-as-point analysis and degree-as-interval analysis are based on the notion of cardinality (Kennedy 2007, 2009).
} 
b. I ate the amount of apples that you ate.

c. I want the amount of apples that Bill received.

In (25a), it is weird that the speaker eats the same apples each day. Similarly, for (25b), it is highly unlikely that the speaker and the addressee eat the same apples. In (25c) the amount of apples refers to some abstract amount, say, 3 kilos of apples.. It is clear that in these existential interpretations, there are two semantic components: an abstract amount/measure, and the objects that instantiate the amount/measure.

Exactly the same pattern is observed for DPs in Chinese. Depending on predicate types, DPs are open to different interpretations. In (26a), san men ke ' $3 \mathrm{CL}$ course' refers to a set of courses, whose cardinality is three. In (26b), san men ke ' 3 CL course' is used as a differential in a DVC construction, which receives an existential interpretation, just like amount does in (25).

\section{(26) a. Zhangsan xuan-le san men ke. \\ Zhangsan take-ASP three CL course \\ 'Zhangsan took three courses.' \\ b. Zhangsan bi Lisi duo xuan-le *(san men ke). \\ Zhangsan BI Lisi DUO take-ASP three CL course \\ 'Zhangsan took three more courses than Lisi did.'}

The standard knowledge of DPs is that they refer to individuals. The semantics of san men ke, for example, can be defined as:

$$
\llbracket \text { san men } k e \rrbracket=\lambda \mathrm{x} . \# \mathrm{x}=3 \wedge * \operatorname{course}(\mathrm{x})
$$

However, san men ke ' 3 CL course' in (26b) does not merely refer to individual courses: it cannot be referred back to by pronouns; nor can it be topicalized. At the same time, san men $k e$ in (26b) does not refer to mere numbers (i.e., cardinality), either, because (26b) does not mean there is a set of numbers that Zhangsan took but Lisi didn't take. On the contrary, san men ke is a "combination" of both the measure and individuals: the speaker specifies a cardinality/measure, which is instantiated by courses. Treating it either as mere individuals or mere numbers would yield the wrong result.

Breaking away from the standard degree-as-point analysis, Scontras (2017) treats degrees as nominalizations of quantity-uniform properties. That is, degrees reference both abstract representation of measurement and the objects that instantiate that measurement. In short, degrees are entity correlates of properties (McNally 2009). Scontras employs the conceptual machinery of "properties" and "kinds" in Chierchia's (1998) to flesh out this idea. Chierchia posits that all first order properties have counterparts in the entity domain such that for any natural property, like the property of being a dog, there corresponds a kind, viz. the dog kind. He defines two semantic operations which relate properties to their entity correlates, and vice versa. The first one is the nominalization process which derives kinds from properties via the "down" operator ", and the second one, the predicativization process, operates in the opposite direction, which retrieves properties from kinds via the "up" operator ${ }^{\text {. The semantics for }}$ these two operators are repeated as below (Chierchia 1998: 349): 


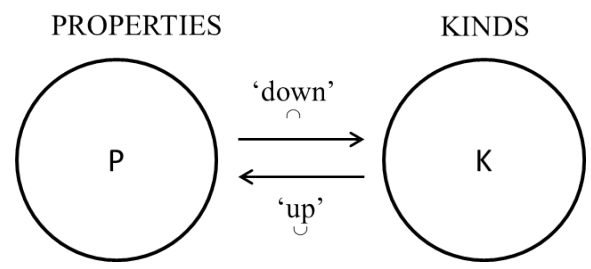

where properties are of type $<\mathrm{s},<\mathrm{e}, \mathrm{t}>>$ and kinds of type $\mathrm{e}$

Example: ${ }^{\mathrm{PANDA}}=\mathrm{k} \quad \sqcup \mathrm{k}=\mathrm{PANDA}$

Chierchia conjectures that for any atomic type, there is a kind counterpart. But what exactly is a kind? The kind PANDA consists of all possible (instantiations of ) pandas ( all the pandas in every possible world). More precisely, the kind is a function from a world to a (typically plural) individual consisting of all the pandas in that world. Correspondingly, to be a realization of the kind PANDA is simply to be a member of the plurality of pandas in a world. This idea echoes with what Cresswell (1976) envisions about the deep connection between individuals and degrees. For him, the degree ' 6 feet tall' is an equivalence class - it contains the plurality of individuals that are 6 feet tall. More specifically, we could think of ' 6 feet tall' as a function from a world to the plurality of 6-foot-tall individuals in that world. This is essentially a Chierchia-style kind. Intensionalizing equivalence classes, we arrive at a Chierchia-style kind:

$$
\llbracket 6 \text { feet man } \rrbracket=\wedge x . \mu_{f}(\mathrm{x})=6 \text { feet } \wedge \operatorname{man}(\mathrm{x})
$$

For every kind $k$, there is a corresponding property satisfied by all and only its realizations. $\bullet k$ is the property counterpart for a kind $k$, where ${ }^{\wedge} P$ is the kind corresponding to a property $P$. If $k$ is a degree-kind of being 6 feet tall, then $k$ is a property of being 6 feet tall (viz. a set of individuals whose height is 6 feet).

(30) $\backsim n \lambda x . \mu_{f}(x)=6$ feet $\wedge \operatorname{man}(x)=\lambda x . \mu_{f}(x)=6$ feet $\wedge$ man $(x)$

For our current purposes, we adopt a simplified version of Scontras' definition of degree (Scontras 2017: 178):

(31) DEGREE $:=\wedge x . \exists k\left[\mu_{f}(\mathrm{x})=\mathrm{n} \wedge \vee k(\mathrm{x})\right]$

(where $\mathrm{k}$ is kind, $\mu_{f}$ is a contextually-specified measure)

The definition in (31) treats degrees analogously to Chierchia-style kinds. Degrees are conceived of as information bundles with four coordinates $\langle\mu, n, k$, $\rangle$ : a measure realized by the measure function $\mu$ (e.g., the kilogram measure, the meter measure, etc.), a value in terms of numbers $n$, a kind $k$, and the Chierchia-style "up" "operator which applies to a kind and returns the property from which the kind is built. In other words, degrees are quantity- and quality-uniform properties, they reference both the abstract measure/amount and the real world objects that instantiate the measure/amount. This new kind of degrees as kinds promises a more motivated account of DVC facts, as to be shown in the rest of this work 


\section{The semantics of DVC sentences}

Having settled on the semantics of degrees, the next task is to determine how this new semantics of degree enters into the semantic composition of DVC sentences to derive the correct truth conditions. This is by no means straightforward.

Anderson and Morzycki (2015) have sketched a semantics for comparatives in a degree-askind analysis. They assume the comparative morpheme -er to have the semantics in (33)

(32) a. Floyd is taller than Clyde is.

b. [DegP -er [CP than Clyde-is tall]] [Floyd tall]

(33) $\llbracket-e r \rrbracket=\lambda \mathrm{k} \lambda \mathrm{s}^{\prime} . \exists k^{\prime}\left[{ }^{\prime} k^{\prime}\left(s^{\prime}\right) \wedge k^{\prime}>_{\mathrm{s}^{\prime}} k\right]$

This semantics is conceptually problematic. Since degrees are kinds, not as real numbers along an abstract scale, it is mysterious how kinds are compared and ordered, as shown by the expression " $k$ ' $>_{\mathrm{s}}$ ' $k$ ". Applying the "up" " operator to retrieve properties from kinds would not help, either, because it is the extensions of a property, not the property itself, are compared and ordered. In other words, the denotation in (33) name entities of the wrong sort. In the following, we provide a semantics for the DVC sentences that discards this semantics based on linear ordering while still conceiving of degrees as kinds.

First, look at the semantics of differential DPs. Take san-ben shu 'three-CL book' as an example. For its syntax, we simply assume that it is some DP-like projection. The "Num-CL" sequence san-ben functions as the modifier to the root noun shu 'book', which is a kind (type $<\mathrm{e}^{\mathrm{k}}>$ ). The degree reading of san-ben shu comes from a covert measure operator $\Delta$ (modeled after Scontras' (2017) amount, read as amount or worth), which connects a kind-denoting term with the measure of the instantiation of that kind. The semantic derivation proceeds as in (35).

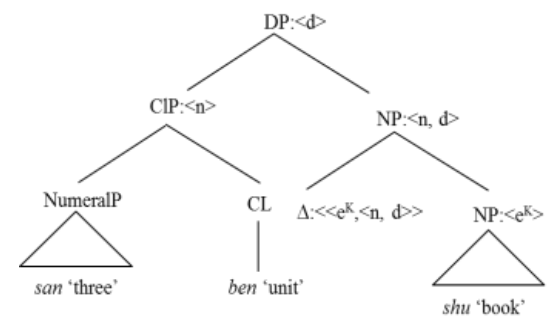

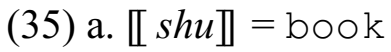

b. $\llbracket \Delta \rrbracket=\lambda \mathrm{k} \lambda \mathrm{n} \lambda \mathrm{d}\left[\mathrm{d}=\lambda \lambda \mathrm{x} . \mu_{f}(\mathrm{x})=\mathrm{n} \wedge \cup \mathrm{k}(\mathrm{x})\right]$

c. $\llbracket \Delta \rrbracket(\llbracket s h u \rrbracket)=\lambda \mathrm{n} \lambda \mathrm{d}\left[\mathrm{d}=\wedge \lambda \mathrm{x} . \mu_{f}(\mathrm{x})=\mathrm{n} \wedge^{\cup} \operatorname{book}(\mathrm{x})\right]$

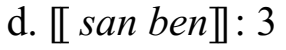

$(<\mathrm{n}, \mathrm{d}>)$

e. [L $[$ san ben $[\Delta s h u]] \rrbracket=\lambda \mathrm{d}\left[\mathrm{d}=\wedge \lambda \mathrm{x} \cdot \mu_{f}(\mathrm{x})=3 \wedge\right.$ book $\left.(\mathrm{x})\right]$

$$
=\wedge \mathrm{x} . \mu_{f}(\mathrm{x})=3 \wedge \cup \mathrm{b} 00 \mathrm{k}(\mathrm{x})
$$

The end result is a degree as nominalized properties. It references both the measure/cardinality $(n=3)$ and the books that instantiate the measure. 
Now consider how the differential DPs (as degree kinds) interact with the structures of DVC sentences in which they participate. To repeat one previous example:

(36) Zhangsan bi Lisi duo du-le *(san ben shu).

Zhangsan BI Lisi DUO read-ASP three CL book

'Zhangsan read three more books than Lisi did.'

Since DVC sentences are about measuring events, we adopt a Kratzerian VoiceP (Kratzer, 1996). The matrix subject Zhangsan start from a low, VoiceP-internal subject position. We also follow Lin (2009) to assume that bi Lisi is adjoined to the VoiceP. No more conceptual machinery is needed to derive the structure of (36).

The crucial part is to settle down the exact semantic of $d u o$ 'more'. Before proceeding, consider the following situation:

(37) Situation: John had a cup of coffee and a donut for this morning. Mary only had a cup of tea.
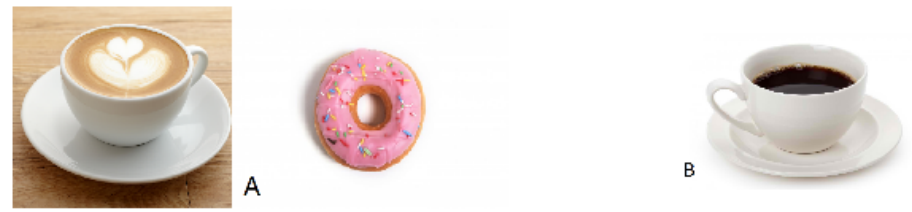

(38) John bi Mary duo chi-le tian-tian-quan.

John BI Mary DUO eat-ASP donut

'John's consumption exceeded Mary's by one donut.'

The scenario depicted in (37) can be felicitously expressed by (38), which means John had one more thing (i.e., a donut) than Mary did This semantics can be expressed by means of difference:

(39) Difference: A is different from $\mathrm{B}$ with respect to donut (x) such that $\mathrm{A}$ had $\mathrm{x}$ but $\mathrm{B}$ did not $\Rightarrow$ In terms of what $A$ and $B$ had, $A$ had $x$ but $B$ did not $\Rightarrow A$ exceeded $B$ by having $\mathrm{x}$.

Obviously, a difference-based analysis entails the A-not-A analysis (Schwarzschild, 2008). We assume that the major semantic function of duo 'more' or shao 'less' in DVC sentences is to express the difference between two individuals $x$ and $y$ with respect to a certain property (or its kind counterpart $k$ ). In the formal literature, there have been some proposals that take comparative morphemes as difference functions (cf. Kennedy and McNally, 2005; Svenonius and Kennedy, 2006; Kennedy and Levin, 2008, among others). In standard degree-based semantics, a difference function is a measure function to measure the degree to which two objects diverge relative to a scalar dimension (Grano and Kennedy, 2012: 235-238). We extend the difference function from the domain of degrees as points to the domain of degrees as kinds. The difference function-based lexical entry of duo is defined in (40): 
(40) $\llbracket d u o \rrbracket=\lambda \mathrm{P}_{<\mathrm{v}, \mathrm{et}} \lambda \lambda \mathrm{d} \lambda \mathrm{x}_{\mathrm{e}} \lambda \mathrm{y}_{\mathrm{e}} \lambda \mathrm{e}_{\mathrm{v}} \cdot \mathrm{P}(\mathrm{e})(\mathrm{v} \mathrm{d}) \stackrel{\mathrm{x}}{\mathrm{g}}(\mathrm{y})$

As shown in (40), duo takes five arguments: a predicate $\mathrm{P}$, a target of comparison y, a standard of comparison $\mathrm{x}$, a degree $\mathrm{d}$, and an event e. In prose, (40) states that an individual y is different from $\mathrm{x}$ with respect to $\mathrm{P}$ relative to some measure $\mathrm{d}$ such that $\mathrm{y}$ holds of $\mathrm{P}$ at $\mathrm{d}$ but $\mathrm{x}$ does not. Actually, this semantics entails an A-not-A analysis.

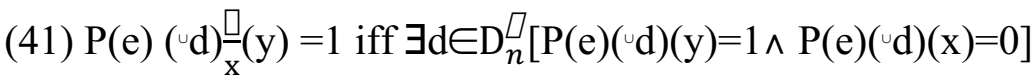

The semantic composition DVC sentences becomes straightforward on this analysis. To illustrate, consider (42) below:

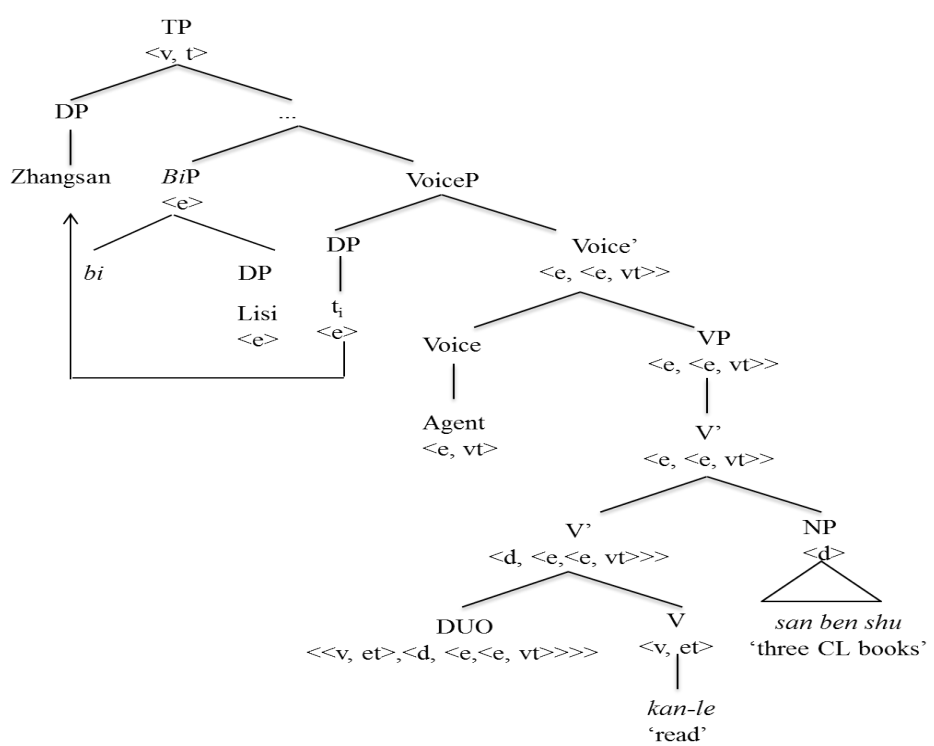

The step-by-step semantic derivation is provided as in (43):

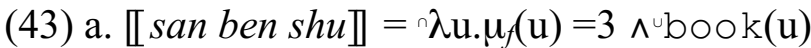

b. $\llbracket d u-l e \rrbracket=\lambda \mathrm{e} \lambda \mathrm{z}$. $\operatorname{read}(\mathrm{e}) \wedge \operatorname{Theme}(\mathrm{e})=\mathrm{z}$

c. $\llbracket d u o \rrbracket=\lambda \mathrm{P}_{<\mathrm{v}, \mathrm{et}}>\lambda \mathrm{d} \lambda \mathrm{x}_{\mathrm{e}} \lambda \mathrm{y}_{\mathrm{e}} \lambda \mathrm{e}_{\mathrm{v}} \cdot \mathrm{P}(\mathrm{e})(\mathrm{d}) \underset{\mathrm{x}}{\stackrel{\square}{\square}(\mathrm{y})}$

d. $\llbracket d u o d u-l e \rrbracket=\lambda \mathrm{d} \lambda \mathrm{x}_{\mathrm{e}} \lambda \mathrm{y}_{\mathrm{e}} \lambda \mathrm{e}_{\mathrm{v}} \cdot \operatorname{read}(\mathrm{e})(\mathrm{d}){ }_{\mathrm{x}}^{\square}(\mathrm{y})$

e. 【duo du-le san ben shu $\rrbracket$

$$
=\lambda \mathrm{x}_{\mathrm{e}} \lambda \mathrm{y}_{\mathrm{e}} \lambda \mathrm{e}_{\mathrm{v}} \cdot \operatorname{read}(\mathrm{e})\left(\mathrm{un} \lambda \mathrm{u} \cdot \mu_{f}(\mathrm{u})=3 \wedge \text { book }(\mathrm{u})\right)_{\mathrm{x}}^{\square}(\mathrm{y})
$$

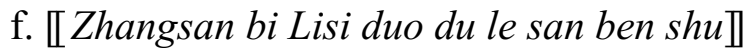

$$
=\lambda \mathrm{e}_{\mathrm{v}} \cdot \operatorname{read}(\mathrm{e})\left(\operatorname{vn}_{\lambda} \mathrm{u} \cdot \mu_{f}(\mathrm{u})=3 \wedge \cup \mathrm{book}(\mathrm{u})\right)_{\mathrm{LS}}^{\square}(\mathrm{ZS})
$$

(44) $\llbracket$ zhangsan bi Lisi duo du le san ben shu $=1$ iff $\exists \mathrm{d} \in \mathrm{D}_{n}^{\square}[\mathrm{P}(\cup \mathrm{d})(\mathrm{ZS})=1 \wedge \mathrm{P}(\mathrm{d})(\mathrm{LS})=0]$

$$
=\exists \mathrm{x}\left[\mu_{f}(\mathrm{x})=3 \wedge^{\bullet} \mathrm{book}(\mathrm{x}) \wedge \operatorname{read}(\mathrm{x})(\mathrm{ZS}) \wedge \neg \operatorname{read}(\mathrm{x})(\mathrm{LS})\right]
$$


Overall, (44) says that "Zhangsan bi Lisi duo du-le san ben shu" is true iff there is some instantiation $x$ of the book kind whose cardinality is 3 such that Zhangsan read $x$ but Lisi did not read (the same thing).Needless to say, this semantics delivers the right truth conditions.

\section{Explaining the facts}

The present analysis garners one immediate advantage: Since kinds can be freely turned into properties via predicativization, with the familiar Derived Kind Predication (DKP), this analysis nicely captures the double behaviors of DPs whereby they reference individuals and reference degrees as kinds at the same time ( cf., (26a) and (26b)). ${ }^{6}$ The present analysis also answers the challenges that DVC sentences pose for the standard semantics. We have shown before that differential DPs in DVC sentences do not manifest the full range of properties associated with individual-denoting DPs. For example, differential DPs in DVC sentences cannot be referred back to by pronouns or empty categories, while they can be referred back to by the degree/kind modifier na'me 'that such'. To repeat one previous example:

$\begin{array}{ccllcll}\text { (45) Zhangsan bi } & \text { Lisi } & \text { duo } & \text { du-le } & \text { [liang ben } & \text { xiaoshuo }]_{\text {i }} \\ \text { Zhangsan bi } & \text { Lisi } & \text { DUO } & \text { read-ASP } & \text { two CL } & \text { novel } \\ \text { ??/* }[\text { Tamen } / e]_{\mathrm{i}} & \text { dou } & \text { hen } & \text { haokan. } & & & \\ \text { they/e } & \text { DOU } & \text { very } & \text { interesting } & & & \end{array}$

This is expected on the present account. Differential DPs denote degrees, and degrees have a different semantics than individuals, and this is why they cannot be referred back to by pronouns/empty categories in DVC sentences. This is further entrenched by the fact they can be referred back to the degree modifier na'me, as shown before.

Another challenge is why differentials are obligatory in DVC sentences. According to Li (2009, 2015a), differential DPs in DVC sentences "indicate at what taxonomic level a mapping relation is established." More specifically, Li argues that certain differential DPs in DVC sentences encode taxonomic information that is necessary for the semantic computation of those sentences. She claims that this extra taxonomic requirement lends support to separating such differential DPs from degree-denoting MPs.

We agree with Li's idea that the standard degree-as-point analysis fails to capture the taxonomic information in differential DPs. But her objection should not apply to the present analysis. On the present account, degrees are quantity- and quality-uniform properties, which means they have two semantic components: besides the measure component, they have another component that contributes properties. Taxonomic information in the different DP is maintained in the present analysis. For example, Jane Eyre he Pride and Prejudice 'JE and PP' and Little Women he Wuthering Heights 'LW and WH' are two distinct pluralities and should not be confused with each other.

This idea provides a natural explanation for the obligatory status of differential DPs in DVC sentences, and relatedly, it also helps reveal what regulates between degrees as kinds and degrees as points. Consider the contrast between (46a) and (46b) below. Recall one essential

\footnotetext{
${ }^{6}$ Due to limitation of space, we have to leave the details aside. Interested readers can consult Chierchia (1998) for details about the shifting between individuals and kinds.
} 
contribution of the gradable adjectives is to provide the dimension along which a scale structure is formed (Kennedy and McNally 2005). The example in (46a) is uninterpretable out of the blue. Lacking an adjective to supply a proper dimension, "three meters" in (46a) is unspecified: it is not clear what it measures (width, length, or height?).

(46) a. *Zhe zhang zhuozi san mi.

Dem CL table three meters

b. Zhe zhang zhuozi san mi chang.

Dem CL table three meters long

'This table is three meters long./ The length of this table is three meters.'

In our analysis, neither duo/shao nor the verbal predicate in the DVC construction provides the necessary dimension for comparison. It falls on the differential phrase to supply the dimension information necessary for the comparison to be facilitated. Take (47) for example. (47a) lacks a proper dimension and sounds unnatural. Adding the differential phrase san gongli 'three kilometers' would supply the dimension of distance, and adding liang ge xiaoshi 'two hours' would supply the dimension of temporal duration. When the verbal predicate is transitive, the differential phrase has the additional function of serving as the object of the verb. Therefore, like Li, we conclude that differential phrases are obligatory in DVC sentences because they provide the dimension information needed to make the comparison meaningful.

(47) a. *Zhangsan bi Lisi duo pao le.

Zhangsan BI Lisi DUO run ASP

b. Zhangsan bi Lisi duo pao-le san gongli / liang ge xiaoshi. Zhangsan BI Lisi DUO run-ASP three kilometers / two CL hours

'Zhangsan ran three kilometers/two hours more than Lisi did.'

On the present account, differential DPs are obligatory in DVCs because they provide the sortal information needed to establish the dimensions of comparison. By contrast, in ACs, because gradable adjectives already contain the information about the dimensions for comparison, differentials become optional. The variation between ACs and DVCs can thus be reduced to how the dimensions for comparison are established, which can be ultimately couched in a theory involving some independently motivated principle of economy (cf., Chierchia's (1998) Blocking Principle and Kennedy's (2007) Interpretive Economy). We leave this topic for future research.

\section{Conclusion}

This article reexamines the Differential Verbal Comparative (DVC) construction in Mandarin Chinese. DVCs exhibit some peculiar properties: (i) obligatory differentials, and (ii) differentials taking the forms of DPs. Li (2015a) claims that the DVC construction is amenable to a mapping-based semantics that compares the individuals in two sets, rather than the cardinalities in two sets. This article takes issue with this degreeless, mapping-based analysis on the ground that the differential DPs in DVC sentences do not manifest the full range of properties of individual-denoting DPs in non-comparative contexts. Building on recent proposals on the ontology of degrees (Anderson and Morzycki, 2015; Scontras 2017), 
this article proposes that a degree is the entity correlate of a property that is formed on the basis of a measure, akin to Chierchia-style kind. We demonstrate how this new kind of degree, plus a difference-based semantics for comparatives, nicely explains a wider range of empirical data concerning DVCs and is an improvement over the previous degree-as-kind analysis such as Anderson and Morzycki (2015).

\section{References}

Anderson, C. and M. Morzycki (2015). Degrees as kinds. Natural Language and Linguistic Theory 33(3): 791-828.

Beck, S. (2012). Comparison constructions. In Maienborn, Claudia et al. (eds.), Semantics: An International Handbook of Natural Language Meaning (Vol. 2), 1341-1389. Berlin and New York: Mouton de Gruyter.

Beck, S. et al. (2004). Parametric variation in the semantics of comparison: Japanese vs. English. Journal of East Asian Linguistics 13: 289-344.

Bhatt, R. and S. Takahashi (2011). Reduced and unreduced phrasal comparatives. Natural Language \& Linguistic Theory 29 (3): 581-620.

Castroviejo, E. and M. Schwager (2008). Amazing DPs. In T. Friedman and S. Ito (Eds.), SALT XVII, pp. 176-193. Ithaca, NY: Cornell University.

Chierchia, G. (1998). Reference to kinds across languages. Natural Language Semantics 6: 339-405.

Cresswell, M. J. (1976). The semantics of degree. In B. H. Partee (Ed.), Montague Grammar, pp. 261-292. NY: Academic Press.

Erlewine, M. (In press). Clausal comparison without degree abstraction in Mandarin Chinese. Natural Language and Linguistic Theory.

Grosu, A. and Landman, F. (1998). Strange relatives of the third kind. Natural Language Semantics 6: 125-170.

Grano, T. (2012). Mandarin hen and Universal Markedness in gradable adjectives. Natural Language \& Linguistic Theory 30(2): 513-565.

Grano, T. and C. Kennedy (2012). Mandarin transitive comparatives and the grammar of measurement. Journal of East Asian Linguistics 21: 219-266.

Heim, I. (1985). Notes on comparatives and related matters. Unpublished ms., University of Texas at Austin.

Heim, I. (1987). Where does the definiteness restriction apply? Evidence from the definiteness of variables. In Reuland, E. and ter Meulen, A. (Eds.), The Representation of (In)definiteness, pp. 21-42. Cambridge, MA: The MIT Press.

Kennedy, C. (2007). Vagueness and grammar: the semantics of relative and absolute gradable predicates. Linguistics and Philosophy 30: 1-45.

Kennedy, C. (2009). Modes of Comparison. In M. Elliot et al. (eds.), Proceedings of CLS 43.

Kennedy, C. and B. Levin (2008). Measure of change: The adjectival core of degree achievements. In L. McNally and C. Kennedy (Eds.), Adjectives and Adverbs: Syntax, Semantics and Discourse, pp. 156-182. Oxford: Oxford University Press.

Kennedy, C. and L. McNally (2005). Scale structure, degree modification, and the semantics of gradable predicates. Language 81(2): 345-381.

Kratzer, A. (1996). Severing the external argument from its verbs. In Johan Rooryck and Laurie Zaring (eds.), Phrase Structure and the Lexicon. Dordrecht: Kluwer.

Li, X. (2009). Degreeless Comparatives. Ph.D. dissertation. Rutgers University. 
Li, X. (2015a). Degreeless comparatives: the semantics of differential verbal comparatives in Mandarin Chinese. Journal of Semantics 32:1-38.

$\mathrm{Li}, \mathrm{X}$. (2015b).The ingredients of comparison: The semantics of the excessive construction in Japanese. Semantics and Pragmatics 8: 1-38.

Lin, J. (2009). Chinese comparatives and their implicational parameters. Natural Language Semantics 17: 1-27.

McNally, L. (2009) Properties, entity correlates of properties, and existentials. In A. Giannakidou and M. Rathert (Eds.), Quantification, Definiteness, and Nominalization, pp. 163-187. Oxford: Oxford University Press.

Rett, J. (2014). The polysemy of measurement. Lingua 143: 242-266.

Schwarzschild, R. (2008). The semantics of comparatives and other degree constructions. Language and Linguistics Compass 2(2): 308-331.

Schwarzschild, R. and K. Wilkinson (2002). Quantifiers in comparatives: a semantics of degree based on intervals. Natural Language Semantics 10: 1-41.

Scontras, G. (2017). A new kind of degree. Linguistics and Philosophy 40: 165-205.

von Stechow, A. (1984). Comparing semantic theories of comparison. Journal of Semantics 3: $1-77$.

Sudo, Y. (2015). Hidden nominal structures in Japanese clausal comparatives. Journal of East Asian Linguistics 24: 1-51.

Svenonious, P. and C. Kennedy (2006). Northern Norwegian degree questions and the syntax of measurement. In M. Frascarelli (Ed.), Phases of Interpretation, pp. 133-161. Berlin: Mouton de Gruyter.

Xiang, M. (2005). Some Topics in Comparative Constructions. Ph.D. dissertation in linguistics. Michigan State University. 